\title{
EL DERECHO A LA SALUD EN COMUNIDADES INDÍGENAS DEL ESTADO DE CHIAPAS
}

\author{
Iliana Amoroz Solaegui \\ iliana.amoroz@gmail.com \\ Universidad Autónoma Metropolitana, Xochimilco \\ México
}

\section{RESUMEN}

Desde la perspectiva de los derechos humanos se analizan las políticas públicas en salud, el cumplimiento efectivo del derecho a la salud por parte del Estado hacia las actuales condiciones de salud y el acceso a los servicios de salud de los pueblos originarios en las regiones Altos, Norte y Selva del estado de Chiapas.

Frente al desdibujamiento del Estado y los magros resultados de las políticas públicas en salud, los pueblos indígenas se enfrentan cada día al intento de acceder a los servicios de salud, a la subordinación y discriminación, condicionados por su pertenencia cultural, lingüística o política.

Este trabajo muestra la grave situación de salud que viven en la actualidad los pueblos originarios ante un sistema de salud cada vez más deficiente e ineficaz. Asimismo comenta las posibilidades de la existencia de mecanismos que hagan exigible y justiciable el derecho a la salud por parte de la población y del Estado, y las demandas y alternativas que se han escuchado desde los pueblos, particularmente en relación con la salud donde los derechos fundamentales para ser efectivos tienen que adquirir un sentido más operacional para la gente y cuyo reto es político.

Palabras clave: pueblos indígenas, derechos humanos, derecho a la salud, políticas públicas. 


\section{SUMMARY}

Public health policies and the effective fulfillment on the part of the State of the right to health are analyzed from the human rights perspective with respect to the current health conditions and access to health services of the native peoples in the High Plains, North, and Forest (Altos, Norte, and Selva) regions of the state of Chiapas.

In the context of the fading State and the meager results of public health policies, the indigenous peoples in their attempts to access health services are faced with subordination and discrimination, conditioned by their cultural, linguistic or political identity.

This work demonstrates the grave health situation lived today by the indigenous peoples in the context of an increasingly deficient and ineffective health system. The possibilities of existence of mechanisms to make the right to health demandable and legally enforceable by the population and the State are discussed, as well as the demands and alternatives heard from the indigenous peoples, in particular in relation to health, in which the fundamental rights, in order to be effective, must acquire a more operational meaning for people. The challenge is political.

Key words: indigenous peoples, human rights, right to health, public policies. 


\section{INTRODUCCIÓN}

El tema de la salud desde la perspectiva de los derechos humanos, DDHH, ha cobrado mayor relevancia en los últimos años. El derecho a la salud se encuentra señalado en los principales estándares internaciones de derechos humanos, particularmente es ubicado dentro de los derechos económicos, sociales y culturales, DESC.

El derecho a la salud en comunidades indígenas adquiere una relevancia y característica importante al conjugarse una serie de elementos que han hecho que estos pueblos originarios sean vulnerados social, económica y políticamente, en condiciones de desigualdad e inequidad en relación con el resto de la población no indígena, lo que ha conllevado a un grave discriminación y violaciones a sus derechos.

Este artículo pretende sólo mostrar, a través de la experiencia de trabajo en la promoción del derecho a la salud y de su exigibilidad y justiciabilidad en comunidades indígenas del estado de Chiapas, durante mi participación en una organización no gubernamental, la grave situación de salud que viven actualmente los pueblos ante un sistema de salud cada vez más deficiente e ineficaz. Las posibilidades de la existencia de mecanismos que hagan exigible y justiciable el derecho a la salud por parte de la población y del Estado; las demandas y alternativas que se han escuchado desde los pueblos, particularmente en relación con la salud.

Por un lado, se plantea el análisis de la situación del cumplimiento del derecho a la salud por parte del Estado a través de las instituciones públicas de salud y el acceso a los servicios de salud de la población indígena en las regiones Altos, Norte y Selva del estado de Chiapas, donde se desarrollan los pueblos tseltal, tsotsil, ch'ol, tojolabal, chuj, kaqchikel y k'anjob'al, a través de algunas reflexiones colectivas sobre el derecho a la salud que permitieron tener un diagnóstico en estas regiones; también se documentan casos de violaciones del derecho a la salud y el trabajo con promotores de salud en la capacitación del derecho a la salud; por otro lado, y a manera solo de acercamiento, se plantea la necesidad de regresar la mirada a los procesos y alternativas que los pueblos han construido para la atención a la salud de la población, procesos organizativos para garantizar la atención primaria de la salud, teniendo como referente cercano al Ejército 
Zapatista de Liberación Nacional, EZLN, que dentro de su propuesta de autonomía ha incluido el Sistema Autónomo de Salud Zapatista, SASZ, como una forma local de organización. ${ }^{1}$

Finalmente, decir que quizá lo empírico de este trabajo tenga que ver con que los temas aquí relacionados: salud, derecho a la salud y pueblos indígenas, son de análisis relativamente recientes en el medio académico, o han sido investigados por separado, tanto desde la Antropología jurídica, la Antropología médica, como desde la perspectiva de los derechos humanos, así como desde el Derecho. Este trabajo intenta abonar al debate.

\section{EL CONTEXTO DE LA GLOBALIZACIÓN: LA SALUD COMO MERCANCÍA}

Las políticas neoliberales, en el marco de la globalización económica, han alcanzado el campo de la salud y han sido en buena medida responsables de la aceleración que vemos en los niveles de pobreza, muertes prevenibles, disparidad, exclusión, desempleo, marginación, alienación, degradación del medio ambiente, explotación, corrupción, violencia y conflictos étnicos y religiosos (Schuftan 2007: 80).

Bajo esta lógica, en México se ha optado por formas no explicitas de privatización en todas las instituciones de salud del sistema, aumentando la inequidad entre la población y mercantilizando la atención a la salud (Eibenschutz 2007: 8). Actualmente los sistemas de salud en México y en América Latina viven un proceso de desmantelamiento de los servicios de salud, a través de la reducción de presupuestos para la implementación de programas; la focalización de subsidios a la población pobre a través de un mecanismo de prepago; disminución del gasto público; modificaciones legales en los sistemas públicos de salud, como el Instituto Mexicano de Seguridad Social, IMSS, el Instituto de Seguridad y Servicios Sociales de los Trabajadores del Estado, ISSSTE, y en programas que cada vez más reducen las acciones de atención a la salud hacia la población. Todo lo cual, desde la perspectiva de los derechos humanos, se traduce en un grave retroceso en la garantía del derecho a la salud de la población. 
Estas políticas neoliberales en salud han sido orientadas por el Fondo Monetario Internacional, FMI, y el Banco Mundial, BM, mismos que han sostenido como estrategias la focalización, la descentralización y la privatización de los servicios sociales (Laurell 1994: 2). En México, desde 1995 y después de la entrada del Tratado de Libre Comercio de América del Norte, TLCAN, las repercusiones de esta política y sus estrategias se han reflejado con mayor intensidad.

\section{EL SISTEMA DE PROTECCIÓN SOCIAL EN SALUD: SEGURO POPULAR}

Una modalidad de esta política es el Sistema de Protección Social en Salud, llamado Seguro Popular, SP, que se puso en marcha desde el año 2001, el cual incluye una modalidad de prepago que suspende el servicio cuando se agota el plazo contratado. El programa no prevé mecanismos que contribuyan a abatir el rezago de infraestructura y servicios, favoreciendo así la inequidad entre las entidades federativas al implementar mecanismos de asignación presupuestal federal proporcionales a los recursos destinados por cada estado.

En 2003 se aprobó la reforma a la Ley General de Salud, LGS, que incorpora la protección social en salud como materia de salubridad general a través de la creación formal del esquema de financiamiento - Sistema de Protección Social en Salud, SPSS - ; y el programa que lo operaría, el Seguro Popular, SP, trazaría las reglas generales de financiamiento y prestación de servicios para el nuevo esquema. Este renovado marco legal sentaría bases para dar contenido al derecho a la salud en México — dentro del discurso de la política internacional de protección universal - al menos formalmente (Pérez 2010: 121).

Sin embargo, en este sentido Laurell (2010) señala que:

...el aseguramiento «universal» se ha planteado como un objetivo de corto o mediano plazo en la mayoría de los países de América Latina durante la última década, y generalmente se ubica dentro de la noción de «protección social». ...ningún seguro de salud ha logrado el propósito de universalidad. ...por otra parte se observa que en muchos países el aseguramiento en salud ha resultado en una nueva dinámica de inclusión-exclusión y en vez de contrarrestar la fragmentación de los sistemas de salud, la ha incrementado (Laurell 2010: 15). 
Este programa ha sido cuestionado y señalado como una regresión en materia de derecho a la salud en México a través de la violación de los principios de gratuidad y universalidad reconocidos constitucionalmente. ${ }^{2}$

\section{EL DERECHO A LA SALUd EN MÉXICO EN EL MARCO DE LOS ESTÁNDARES INTERNACIONALES DE DERECHOS HUMANOS}

Paralelamente a este proceso de globalización y crisis neoliberal productora de desigualdad, exclusión e inequidad, desde la perspectiva de los derechos humanos la salud ha tenido otra dimensión, avances y relevancia en los últimos años desde el planteamiento del derecho a la salud, y cuyo contenido está plasmado en diversas declaraciones, convenios y pactos de derechos humanos en el ámbito internacional desde mediados del siglo XX. El derecho a la salud pertenece a los derechos de segunda generación, los derechos económicos, sociales y culturales, cuyo estándar máximo es el Pacto Internacional de Derechos Económicos, Sociales y Culturales, PIDESC (1966), del Sistema de Naciones Unidas, ONU.

El Comité de Derechos Económicos Sociales y Culturales, CDESC, organismo también adscrito a ONU, fue creado para la promoción y vigilancia del cumplimiento del Pacto de Derechos Económicos, Sociales y Culturales, PIDES, que en 2000 amplía, a través de Observaciones Generales, los derechos reconocidos en cada uno de los artículos que conforman PIDESC, como son el derecho a una vivienda adecuada, a la alimentación, a la salud, al agua, a la educación, entre otros.

La Observación General número 14 es la que corresponde al artículo 12 sobre «el derecho al disfrute del más alto nivel posible de salud», donde se define el contenido mínimo esencial del derecho a la salud que se refiere al acceso universal a bienes y servicios de salud, a medicamentos esenciales, a asistencia médica y sanitaria preventiva y curativa, a un plan nacional de salud pública, a la provisión de seguro y de servicios de salud para quienes carecen de recursos, a la educación e información en salud y a la cooperación internacional. Señala también los elementos esenciales mínimos del cumplimiento del derecho a la salud en cuanto a disponibilidad, 
accesibilidad — no discriminación, accesibilidad física, accesibilidad económica, acceso a la información—, aceptabilidad y calidad de los servicios de salud.

A escala nacional, en la Constitución Política de los Estados Unidos Mexicanos, en su artículo 4 hace referencia al derecho a la protección de la salud, también en leyes subsecuentes como la Ley General de Salud, donde se incorpora una serie de referencias sobre el derecho a la protección de la salud, de ésta a su vez se desprenden las leyes estatales de salud.

Sin embargo, los mecanismos de exigibilidad y justiciabilidad de este derecho que puedan activarse ante una violación de la ley no están establecidos con claridad. El derecho a las salud, hasta mediados de 2007, era un tema que no había sido explorado en lo que se refiere a litigio, lo que hay en la actualidad en cada institución es la posibilidad de realizar trámites y quejas, que en el mejor de los casos llegan a ser faltas administrativas y con resultados procedimentales. Existe como una opción la Comisión Nacional de Arbitraje Médico, Conamed, que de llegar a ser conocida dicha instancia por la población es donde podría acudir a poner una queja.

La Comisión Nacional de Derechos Humanos, CNDH, ha sido una de las opciones para las personas que han sido víctimas de alguna violación del derecho a la salud, es significativo que para 2005 el primer lugar en quejas en esta institución fue en contra del Instituto Mexicano del Seguro Social, IMSS, con 803 escritos en contra, por arriba de las quejas presentadas contra la Procuraduría General de la República, PGR. El sexto lugar, con 217 quejas, lo ocupó el Instituto de Seguridad y Servicios Sociales de los Trabajadores del Estado ISSSTE, por debajo de la PGR, la Comisión Federal de Electricidad, CFE, y el Instituto Nacional de Migración, INM; es decir, de los derechos sociales es el que más ha sido violado.

En el ámbito internacional, en las instancias de Justicia, la situación de protección y exigibilidad de los derechos económicos, sociales y culturales en relación con los derechos civiles se da de manera asimétrica. Sin embargo, ante la Comisión Interamericana de Derechos Humanos, $\mathrm{CIDH}$, ya se han empezado a recibir denuncias 
por violación del derecho a la salud, sobre todo en cuanto a tratamiento y atención a enfermos de Síndrome de Inmunodeficiencia Adquirida, Sida, y la Seguridad Social (Echeverri 2006: 86).

En el trabajo realizado en el Centro de Capacitación en Ecología y Salud para Campesinos, CCESC, y junto con la Comisión Mexicana de Promoción y Defensa de los Derechos Humanos, CMDPDH, A. C., se llevó ante CIDH una petición de medidas cautelares para cinco enfermos de tuberculosis en el estado de Chiapas, y la búsqueda de 144 enfermos detectados a través de un estudio realizado en 2002 por El Colegio de la Frontera Sur, Ecosur, en la región Altos; sin embargo, la respuesta a esta petición fue burocráticamente lenta en relación con la gravedad de los enfermos, más en lo relacionado con sus condiciones culturales específicas.

Queda visible la distancia que existe entre los avances del contenido jurídico del derecho a la salud plasmado en pactos, convenios y constituciones, y las posibilidades reales del cumplimiento, respeto y garantía de dicho derecho hacia la población, más grave aun si consideramos el contexto de los efectos de las reformas neoliberales y sus consecuencias en la profundización de las inequidades y desigualdades en salud y acceso a los servicios para la población, principalmente en contextos rurales e indígenas.

En este sentido la participación del Estado es central, ya que juega un papel importante en la garantía del derecho a la salud a través del diseño y la implementación de políticas sociales, así como en la creación de mecanismos para hacer exigible y justiciable este derecho. En esto la participación activa de la población y la sociedad civil organizada se vuelve necesaria.

De aquí la importancia de resaltar y realizar trabajos más empíricos, locales y comunitarios, que permitan abordar e identificar las posibilidades de la acción social y política para la exigibilidad del derecho a la salud como vías para presionar la obligatoriedad jurídica del Estado, que él mismo ha asumido al firmar y ratificar los 
estándares internacionales de derechos humanos, como el Pacto Internacional de Derechos Económicos, Sociales y Culturales, donde se encuentra el derecho a la salud.

En México, en 2008 se dio el primer caso que sienta jurisprudencia sobre el derecho a la salud contemplado en los estándares internacionales de derechos humanos, a través de las denuncias y demandas colectivas que desde 2007 realizaron los indígenas $\mathrm{Na}$ Savi de la comunidad Mini Numa, en el municipio de Metlatónoc, ubicada en la región de la Montaña del estado de Guerrero, y quienes ante las adversas condiciones de salud y acceso a los servicios que viven, a través de la Comisión de Defensa de los Derechos Humanos del estado de Guerrero, Coddehum, solicitaron al gobierno guerrerense medidas cautelares ante las condiciones de salud que padecían. Al tener una respuesta negativa, decidieron solicitar un recurso de amparo ante el juzgado de distrito en Chilpancingo, en contra de la determinación del secretario de salud del Estado, mismo que les fue otorgado. ${ }^{3}$

\section{ESTÁNDARES INTERNACIONALES DEL DERECHO A LA SALUD}

México ha ratificado diversos tratados, convenios y protocolos en materia de derechos humanos en los que se ha comprometido a proteger, promover, respetar y garantizar una serie de derechos civiles, políticos, económicos, sociales y culturales que contienen estos estándares, como el Pacto de Derechos Económicos, Sociales y Culturales (PIDESC 1966) que en su artículo 12 se refiere al derecho a la salud y especifica:

1. Los Estados Partes en el presente Pacto reconocen el derecho a toda persona al disfrute del más alto nivel posible de salud física y mental.

2. Entre las medidas que deberán adoptar los Estados Partes a fin de asegurar la plena efectividad de este derecho, figurarán las necesarias para:

a) La reducción de la mortinatalidad y de la mortalidad infantil, y el sano desarrollo de los niños;

b) El mejoramiento en todos sus aspectos de la higiene del trabajo y medio ambiente;

c) La prevención y tratamiento de las enfermedades epidémicas, profesionales y de otras índole y la lucha contra ellas;

d) La creación de condiciones que aseguren a todos asistencia médica y servicios médicos en caso de enfermedad. 
El PIDESC, en el año 2000, fue actualizado a través de una serie de observaciones a cada artículo que lo conforman a través del Comité de Derechos Económicos, Sociales y Culturales (CDESC 2000). La observación número 14 es la que corresponde al artículo 12 de PIDESC, que establece:

El derecho a la salud está estrechamente vinculado con el ejercicio de otros derechos humanos ... en particular el derecho a la alimentación, a la vivienda, al trabajo, a la educación, a la dignidad humana, a la vida, a la no discriminación, a la igualdad, a no ser sometido a torturas, a la vida privada, al acceso a la información y a la libertad de asociación, reunión o circulación. Esos y otros derechos y libertades abordan los componentes integrales del derecho a la salud.

Es decir, que el cumplimiento del derecho a la salud no se reduce a la atención médica, sino que su cumplimiento también está íntimamente relacionado con el ejercicio de otros derechos económicos, políticos, culturales y sociales.

Dentro de las Observaciones también quedan señalados los elementos básicos del cumplimiento del derecho a la salud que los Estados están obligados a garantizar:

Disponibilidad. Cada Estado deberá contar con un número suficiente de establecimientos, bienes y servicios públicos de salud y centros de atención de la salud, así como programas.

Accesibilidad. Los establecimientos, bienes y servicios de salud, deben ser accesibles con las características de no discriminación, accesibilidad física, económica y acceso a la información.

Aceptabilidad. Todos los establecimientos, bienes y servicios de salud, deberán ser respetuosos de la ética médica y culturalmente apropiados.

Calidad. Además de aceptables desde el punto de vista cultural, los establecimientos, bienes y servicios de salud, deberán ser de buena calidad, así como apropiados, desde el punto de vista científico y médico.

El Convenio 169 sobre Pueblos Indígenas y Tribales en Países Independientes establece en sus artículos 24 y 25 :

Artículo 24

1. Los regímenes de seguridad social deberán extenderse progresivamente a los pueblos interesados y aplicárseles sin discriminación alguna. 
Artículo 25

1. Los gobiernos deberán velar porque se pongan a disposición de los pueblos interesados servicios de salud adecuados o proporcionar a dichos pueblos los medios que les permitan organizar y prestar tales servicios bajo su propia responsabilidad y control, a fin de que puedan gozar del máximo nivel posible de salud física y mental.

En el tema de salud existe la Declaración de Alma Ata, documento que en 1978 firmaron 134 gobiernos y 67 organizaciones no gubernamentales y organismos internacionales, que se comprometen a lograr salud para todos en el año 2000. Los principios de los que parte y que asienta esta Declaración son el contenido de lo que se define como Atención Primaria de Salud, APS, comprensiva, integral, universal e incluyente. A treinta años de esta Declaración es claro que no se ha logrado el objetivo.

\section{EXIGIBILIDAD Y JUSTICIABILIDAD DEL DERECHO A LA SALUD}

La exigibilidad jurídica de un derecho implica tener por objetivo que el Estado cumpla con las obligaciones a las que se ha comprometido al ser firmante y ratificante de convenios y tratados en materia de derechos humanos. La justiciabilidad es la posibilidad de reclamar ante un juez o tribunal de justicia el cumplimiento al menos de algunas de las obligaciones que se derivan del derecho (Abramovich y Courtis 2003: $61)$.

Se entiende por derecho social no solo la conducta cumplida por el Estado, sino además la posibilidad de reclamo ante el incumplimiento: que el titular/acreedor esté en condiciones - al menos en alguna medida — de producir mediante una demanda o queja el dictado de una sentencia que imponga el cumplimiento de la obligación generada por su derecho (Abramovich y Courtis 2003: 61). La exigibilidad como proceso político significa el poder incidir en las políticas públicas y programas gubernamentales, así como el impulso a leyes o reformas de leyes, movilizaciones y acciones para promover el ejercicio de los derechos económicos, sociales y culturales.

Se tiene así, por delante, el reto de un trabajo que genere la participación social o comunitaria, es decir, que se pueda exigir un derecho que le corresponde y que puedan 
obtener respuestas a sus demandas de parte de las instituciones públicas, en los diferentes órdenes de gobierno, ya sea municipal, estatal, nacional e internacional.

\section{LA SITUACIÓN DE SALUD EN CHIAPAS}

Chiapas, Guerrero, Oaxaca, Veracruz y Puebla, son los estados que concentran las mayores desigualdades en relación con el cumplimiento del derecho a la salud, de igual manera son los que cuentan con menos servicios de atención y con más población que gana menos de dos salarios mínimos.

En el estado de Chiapas se encuentra la mayoría de los municipios catalogados de alta y muy alta marginación del país: $77 \%$ de los municipios chiapanecos de muy alta marginación son indígenas (Coespo 2000, citado en Sánchez 2007). Es el estado que tiene más bajos Índices de Desarrollo Humano, IDH; comunidades donde se padecen enfermedades prevenibles, tratables y curables como la tuberculosis, la desnutrición en niños menores de 5 años y la muerte materna, situaciones estrechamente relacionadas con sus contextos de pobreza, inequidad, desigualdad y de injusticia; por tanto, donde se dan de manera sistemática violaciones de los derechos humanos de la población.

Es el estado que presenta el mayor rezago en cobertura de los sistemas de salud, principalmente de la Secretaría de Salud, SSA, y el IMSS-Oportunidades, especialmente en las regiones con mayor población indígena. En $80 \%$ de las comunidades chiapanecas existe sólo un médico por 2,370 habitantes, y constituye el estado con menor porcentaje de población cubierta por algún esquema de seguridad social (Sánchez 2007: 293).

El acceso a los servicios de salud, el bajo gasto en salud per cápita, el número de médicos y de camas de hospital por habitante, la escasez de medicamentos, la falta de equipo médico y de hospitales de segundo y tercer nivel, están muy lejos de cubrir las recomendaciones internacionales, situaciones que una vez más ponen en evidencia la inequidad y desigualdad en que se encuentra la población. 


\section{LA SITUACIÓN DE LA SALUD EN LAS REGIONES ALTOS, NORTE Y SELVA}

A través del trabajo en la organización es que pudimos conocer de cerca algunas de las situaciones y demandas de 46 comunidades de las regiones Altos, Norte y Selva, con población indígena tsotsil, tseltal, chó1, tojolabal, k'anjobal, chuj y kaqchikel, que se visitaron con el motivo de la socialización del informe Pueblos excluidos. Comunidades erosionadas. La situación del derecho a la salud en Chiapas, México, donde se vertían los resultados de un estudio basal que habían realizado cuatro años atrás en la región de conflicto dos organizaciones y una institución de investigación (PHR, CCESC, Ecosur 2006).

Las conclusiones se dieron en torno a temas de salud sobre mortalidad materna, desnutrición en niños menos de 5 años y tuberculosis, así como la situación sobre los servicios de salud. Algunos de los resultados que se dieron a conocer fueron los siguientes:

- Mortalidad Materna. Se documentaron 1,223 embarazos durante los dos años previos al estudio, de los cuales ocho, $0.7 \%$, concluyeron en muerte materna. De estas 1,223 mujeres embarazadas $53.9 \%$ recibió atención prenatal por parte de una partera tradicional, PT; 8.6\% recibió atención prenatal tanto de la partera como del personal de salud gubernamental; $29.7 \%$ fue atendido exclusivamente por los servicios de salud gubernamentales y $1.3 \%$ por otro tipo de servicios de salud privados; $2.6 \%$ por agentes no sanitarios — familiares—, y $3.9 \%$ no recibió atención (PHR, CCESC, Ecosur 2006:33).

- De las ocho muertes maternas que se documentaron todas eran mujeres indígenas y murieron principalmente por tres razones: retraso en la decisión de buscar ayuda por falta de dinero. De trasporte y de un apoyo médico en la comunidad. Retraso en llegar al sitio de atención, dos mujeres murieron en el camino y una más esperando transporte. La tercera situación de muerte se debió al retraso en recibir atención y la capacidad para resolver la emergencia por parte de los médicos. Cuatro mujeres murieron en el hospital (PHR, CCESC, Ecosur 2006: 35).

- Desnutrición en niños menores de 5 años. La mayoría de los niños tiene desnutrición crónica. En los niños de uno a cinco años encontramos que más de la mitad tiene este tipo de desnutrición. En el país es diferente, solo uno de cada cuatro niños sufre de desnutrición (PHR, CCESC, Ecosur 2006: 37).

- Tuberculosis Pulmonar en mayores de 15 años. De 29 personas enfermas de tuberculosis que se encontraron, solo 13 habían sido detectadas por los servicios de salud. Una de estas personas 
no había recibido tratamiento. 10 tenía señas de estar enfermas, pero no les había realizado estudios; 4 no había recibido atención médica (PHR, CCESC, Ecosur 2006: 41).

- Sobre la utilización de los servicios de salud, tres de cada diez personas que enfermaron en el mes anterior al estudio no buscaron atención médica. Seis de cada diez buscaron atención en los centro de salud (PHR, CCESC, Ecosur 2006: 44-45).

Durante el proceso de socialización de estos resultados, en 2007 la situación no había cambiado mucho, al contrario, en algunos casos incluso había empeorado, según la percepción de la gente compartida en reuniones comunitarias acordadas. En la mayoría de las comunidades se convocó a todos sus habitantes y se reflexionó sobre la situación del derecho a la salud en sus lugares. Las que siguen son algunas de las situaciones que se encontraron durante los recorridos por las comunidades:

- Manifiestan recibir regaños constantes y malos tratos por parte del personal de salud.

- Se quejan de que constantemente se les amenaza con quitarles los beneficios de programas, principalmente de Oportunidades, si no asisten a la clínica y si no participan en reuniones. De manera específica se mencionó que las mujeres sienten que se les amenaza si no asisten a que se les tome un frotis de cérvix uterino para la prueba de Papanicolau o aceptan métodos de planificación familiar.

- Problemas de comunicación. Se quejan de que no se les explican bien los programas ni las acciones que se realizan. Con gran frecuencia se mencionó la vacunación y la falta de claridad de la población sobre los cambios en el esquema, la frecuencia y la vía de administración de las vacunas.

- Se menciona con mucha frecuencia que no hay traductores y que el personal de salud no sabe la lengua local ni se esfuerza por hacerse entender.

- Falta de medicamentos. Se menciona la falta de medicamentos como una de las mayores deficiencias de los servicios.

- La poca permanencia de los médicos en la localidad. Llegan solo tres o cuatro días a semana, llegan tarde y se van temprano, se mencionó que éstos nunca se encuentran por las tardes y las noches, que es cuando tienen emergencias o cuando se producen las muertes.

- Ocho comunidades no tienen servicio de salud. Son visitadas un día al mes, durante algunas horas por una unidad móvil. La población tiene que desplazarse, algunas a pie, más de dos horas para recibir atención.

- En todas las localidades, la población se interesa mucho por conocer más acerca de sus derechos, así como por saber cómo y adónde recurrir en caso de problemas. ${ }^{4}$ 
El panorama de la situación del derecho a la salud en la regiones indígenas es desolador, aproximadamente $90 \%$ no cuenta con derecho a servicio de salud; existe una gran discriminación por parte del personal médico en las comunidades hacia las personas indígenas y hacia la lengua que hablan; hay una constante falta de medicamentos en los centros de salud y por tanto se ven en la necesidad de comprarlos, invirtiendo más de la mitad de sus ingresos en ello; falta de conocimiento sobre adónde pueden dirigir las demandas y quejas de las situaciones que pasan en su comunidad; existencia de una sola clínica para atender varias comunidades; falta de trasporte para llevar a las clínicas u hospitales a los enfermos, y aparición de enfermedades relativamente nuevas como la hipertensión y la diabetes, otras como infecciones por contaminación del agua y del ambiente, son tan solo algunas de las cotidianas situaciones que narraron vivir las personas que pueblan comunidades indígenas de estas regiones.

\section{TUBERCULOSIS, LA ENFERMEDAD DE LA POBREZA}

La tuberculosis, TB, es una de las enfermedades que pone en evidencia las desigualdades en el acceso a los servicios y la calidad de la atención, ya que es enfermedad evitable, y en su caso diagnosticada, tratada y curada, siempre y cuando se lleve el tratamiento y seguimiento adecuado.

Chiapas ocupa el segundo lugar de morbilidad-mortalidad por tuberculosis después de Veracruz, con un promedio de $8 \%$ de incidencia respecto al total nacional. Es decir, que de cien casos de tuberculosis registrados en México ocho están en Chiapas (Inegi-OPS, 2000-2004).

Entre los principales problemas que implican que Chiapas sea uno de los estados más afectados por TB en el país, se encuentran los relativos a los servicios de salud, esto es, en cuanto al diagnóstico, tratamiento y seguimiento de pacientes con TB. Solo $24 \%$ de las personas que tienen TB es diagnosticado por los servicios de salud. Dos aspectos ayudan a explicar esta situación:

1) El método que se usa para detectar casos — basiloscopia - es vital; dada la falta de equipo y recursos para la toma y procedimiento — falta de capacitación — de muestras de la flema — 
gargajo- de los tosedores existe una alta probabilidad de subregistro de casos, es decir, los resultados de las pruebas dan que una persona no tiene TB, cuando en realidad dicha persona sí está enferma.

2) Una de las causas reconocidas para que no se haya controlado la TB es el problema de actitudes de los médicos, cuando hay en las clínicas de las comunidades, quienes no indagan adecuadamente sobre síntomas respiratorios entre sus pacientes (Sánchez et al. 2008).

Por su parte, se ha estimado que del total de enfermos con TB en zonas de muy alta marginación socioeconómica, como es la región Altos, únicamente 10\% recibe tratamiento. De ese $10 \%$ la mayoría no recibe el método adecuado - cuya duración mínima es de seis meses - debido a una serie de factores que involucran a las personas - por ejemplo, algunos dejan el tratamiento cuando se sienten mejor-y a los propios servicios de salud — falta de seguimiento, falta de abasto de medicamentos, mal trato a los pacientes-. Los casos presentados demuestran lo relativo a estas deficiencias (Sánchez et al. 2008).

Casi una décima parte de los enfermos diagnosticados con tuberculosis pulmonar, TBP, del país se encuentra en Chiapas; no obstante de que algunos investigadores han estimado que el subregistro es mayor a cincuenta por ciento (Sánchez 2002) Chiapas tiene la mayor tasa de mortalidad por tuberculosis.

Como parte de las actividades de la organización se documentaron casos de violación del derecho a la salud a enfermos de TB, se encontraron y dio seguimiento a los siguientes casos:

...se encuentra en mal estado general secundario, ha presentado hemoptisis; presenta una desnutrición moderada, astenia y adinamia. Persiste tos con expectoración abundante y diaforesis nocturna. Estos síntomas se presentan desde hace cinco años aproximadamente, con intensidad variable, pero sin franca mejoría. Su estado le impide trabajar... (hombre, tsotsil, entrevista realizada en la comunidad El Pozo, municipio de Oxchuc, región Altos, Chiapas, marzo, 2008).

...señora con abundante tos productiva de predominio nocturno, diaforesis. Su debilidad propicia infecciones secundarias recurrentes tales como diarreas, infecciones de la garganta. Presenta cuadros febriles frecuentes, dolor torácico. Presenta desnutrición leve y anemia. Su estado 
general le impide realizar sus actividades cotidianas... (mujer, tsotsil, entrevista realizada en la comunidad Jardín Nuevo Edén, municipio de Teopisca, región Altos, Chiapas, enero, 2008).

...después de andar de clínica en clínica me atendieron en una organización civil y me diagnosticaron TB, para darle seguimiento al tratamiento fui a una clínica del IMSS de mi comunidad donde me dijeron que esa enfermedad no la sabían tratar... (hombre, k'anjob'al, entrevista realizada en la comunidad de Nuevo Huixtán, municipio de las Margaritas, región Selva, Chiapas, mayo, 2008).

Ante este panorama en reiteradas ocasiones se ha insistido en la grave situación, a través de centros de investigación y organizaciones no gubernamentales e internacionales en el estado. Una de estas ocasiones fue entre finales de 2007 y principios de 2008 donde se planteó la grave situación de los enfermos con tuberculosis en el estado ante instituciones de salud estatales e incluso nacionales, así como a instancias internacionales, ${ }^{5}$ demandando entonces:

1. Falta de medicamentos antifímicos para el tratamiento.

2. Deficiencias en el diagnóstico y en la atención.

3. Deficiencias en el seguimiento del tratamiento primario que deben llevar los enfermos.

4. Falta de diagnóstico y tratamiento para personas farmacorresistentes.

En el año 2008 estos casos fueron llevados ante la Comisión Interamericana de Derechos Humanos, CIDH, al mismo tiempo de visibilizar la situación de la salud en Chiapas; se inició así el proceso de denuncia de los derechos sociales ante instancias internacionales. Situación que evidenció una serie de violaciones al derecho a la salud, según lo establecido en los estándares internacionales de derechos humanos, cuyo marco de definición del derecho a la salud se refiere al disfrute de toda una gama de facilidades, bienes, servicios y condiciones necesarias para alcanzar el más alto nivel posible de salud; elementos esenciales que deben estar presentes en el cumplimiento del derecho a la salud. Sin embargo tan solo en un padecimiento, por demás grave, se pone en evidencia que no se ha cumplido, respetado y garantizado el derecho a la salud de los enfermos con tuberculosis, o de igual manera el acceso a la protección de la salud según la Constitución. De acuerdo con los elementos mínimos que se deben garantizar para el cumplimiento efectivo y en relación con la tuberculosis, se tienen los siguientes: 
1. Disponibilidad. Los establecimientos, bienes y servicios de salud, ubicados en comunidades indígenas, no cuentan con personal médico y profesional capacitado para el diagnóstico, atención y seguimiento del tratamiento de TB, tampoco cuentan con los medicamentos necesarios, y en muchos casos ni siquiera con vasos para tomar muestras de esputo.

2. Accesibilidad. Los establecimientos, bienes y servicios de salud, principalmente aquellos ubicados en municipios y comunidades indígenas, no son accesibles a toda la población porque se discrimina, se encuentran lejos geográficamente y no se da información clara sobre la enfermedad.

3. Aceptabilidad. Los establecimientos, bienes y servicios de salud, no son respetuosos de las culturas indígenas de donde se localizan, y en muchos casos tampoco son sensibles a los requisitos que implica el trato hacia mujeres.

4. Calidad. No son de calidad, pues no se cuenta con personal capacitado para el diagnóstico, seguimiento y tratamiento de los enfermos de $\mathrm{TB}$, lo que provoca deficiencias técnicas en el diagnóstico.

Las instituciones públicas de salud tampoco están garantizando ni respetando los derechos de los pueblos indígenas del estado, entre ellos los tsotsiles, tseltales, choles, k'anjob'ales, que se localizan en las regiones Altos, Norte y Selva, entre otros, y que de acuerdo con el convenio 169 el Estado debería velar porque se pongan a disposición de los pueblos interesados servicios de salud adecuados.

\section{LOS PROMOTORES DE SALUD COMO SUJETOS PARA LA EXIGIBILIDAD DEL DERECHO A LA SALUD ${ }^{6}$}

Otro de los trabajos que se realizaron en 2008 a través de la organización civil ${ }^{7}$ fue el de la promoción y capacitación en derecho a la salud a promotores de salud de la Red de Promotores y Parteras, Repropar, de la región Selva, que agrupa a tres organizaciones de promotores de salud y se distribuyen en sesenta comunidades y cinco municipios de la regiones Selva y Fronteriza. Los promotores de salud al día de hoy son los encargados de brindar atención primaria de la salud. Fueron formados en la década de 1980, a partir del trabajo en salud por diferentes sectores como la Iglesia, las organizaciones no gubernamentales y las universidades, quienes realizan trabajo en varias regiones del estado, principalmente rurales e indígenas, regiones donde la pobreza y desigualdad son características. 
Promotor de salud es:

... [aquella] persona campesina, casi siempre indígena, la cual ha sido capacitada en habilidades de diagnóstico, tratamiento y medidas preventivas desde la perspectiva de la medicina occidental. Su existencia ha respondido a la necesidad de resolver los problemas más comunes de enfermedad, detectando a tiempo las situaciones de riesgo para referirlas a otro nivel de atención, en aquellos lugares alejados de servicios médicos. Uno de sus quehaceres es la promoción de la salud a través del impulso de acciones preventivas, pero también desde una perspectiva social del proceso salud-enfermedad, hacer del conocimiento de la población que la enfermedad tiene causa profundas que están sustentadas en una situación política injusta (Rivas 1999: 97).

La mayoría de los promotores y promotoras de Repropar son por los más numerosos indígenas tseltales, tsotsiles, indígenas refugiados guatemaltecos chuj, kaqchikel y k'anjob'al, además de mestizos; muchos de ellos fueron de los primeros formados como promotores durante la década de 1980 por instituciones con presencia ya en la región, como el Hospital General de Comitán y el Centro en Ecología y Salud para Campesinos, CCESC, ligado al Instituto Nacional de Nutrición Salvador Zubirán, INNSZ. Muchos de los promotores con los que se trabajó tenían más de 15 años de ejercicio y se habían formado en este proceso, asimismo presentaban un claro panorama de la situación de salud en la región, así como de las políticas sociales.

Simón llegó de Guatemala en 1983 como refugiado. Así habló de ello:

\footnotetext{
No había caminos, clínicas ni hospitales. El hospital de Comitán se encargaba de llevar atención médica a todas las comunidades. La salud de las comunidades se encontraba en una situación bastante crítica. Los refugiados llegaban con hambre y desnutrición. Casi una de cada diez personas moría por las condiciones en las que se encontraban. Había poco comercio, no había electricidad. ${ }^{8}$
}

También se pudieron identificar las características de la relación entre el trabajo de los promotores con las instituciones: 
Desde hace diez años hemos luchado como organización con la Secretaría de Salud, porque desde ese tiempo no reconoce la labor de la promotora o promotor en nuestras comunidades, y como organización hemos puesto nuestras demandas ante autoridades de salud regional y estatal, sobre todo abasto de medicamentos, materiales y equipos. Y nos han dicho que no hay respuesta ante ello. Hay unidades médicas móviles que tienen como ruta visitar cada 15 días a cada comunidad, pero no cumplen con el compromiso, y lo que han hecho las personas es que se amontonan cuando llegan las unidades móviles, y quedan personas sin atender y niños sin vacunar, entonces no es accesible. ${ }^{9}$

Algunas violaciones del derecho a la salud por discriminación:

Negación de la atención médica por ser guatemalteco y/o apariencias de centroamericanos, porque el médico suele decir que está fuera del horario de servicio. Frases como «Espérese hasta las 4», y «ese pinche guatemalteco debe estar en su país no debe estar aquí». A personas de Guatemala les niegan la atención, los discriminan por no ser mexicanos, por ser de Guatemala, por no ser de la misma comunidad, o no tener seguro popular. Humillación a los pacientes, específicamente para los indígenas, porque no tienen con qué defenderse. ${ }^{10}$

Nosotros como asimilados e integrados en el estado de Chiapas, para nosotros no hay un presupuesto de medicamentos, y no hay médicos, eso nos dicen las autoridades. Hemos presentado documentos solicitando medicamento ante autoridades regionales como Guillermo Vilchis Torres, Eugenio Salinas Linares, la respuesta es que como somos guatemaltecos no hay presupuesto para nosotros. Y son 9 comunidades, aproximadamente 2,000 personas. Llevamos un año que no llevan vacuna a los niños, ni la BCG. ${ }^{11}$

Por no hablar español:

Negación a la atención por no hablar el mismo idioma, por no ser mexicano, no hay respuesta a los métodos de planificación familiar, deslindan responsabilidad en la unidad médica, desvalorización como promotor. No hay medicamentos. ${ }^{12}$

Y así muchos, incontables casos que comentan cada uno de los promotores, quienes dan atención en su lengua, a la hora que se necesite, aplican incluso acupuntura que llegaron a aprender en algún curso que recibieron en la clínica de Nuevo Poza Rica, clínica que se construyó con apoyo del Instituto Nacional de Nutrición Salvador Zubirán y que actualmente está a cargo del Centro de Asesoría Medio Ambiente y Defensoría del 
Derecho a la Salud, CAMADDS, A. C. La población confía en ellos. El trabajo directo, constante y de acompañamiento a escala local y comunitaria en la exigibilidad y justiciabilidad del derecho a la salud representa un reto tanto para la población local, en general, como para las organizaciones de la sociedad civil y las instituciones públicas, ya que en la medida de ser ellos los más preocupados e interesados en resolver los serios y graves problemas de salud a los que cotidianamente se enfrentan en la atención a la población, son los promotores de salud sujetos potenciales para el mejoramiento de la atención a la salud y de las graves condiciones de salud de la población.

\section{LA SALUD EN RESISTENCIA. DEMANDAS Y PROPUESTAS DESDE LOS PUEBLOS}

Una salud libre hace un corazón más rebelde Caracol que habla para todos, Roberto Barrios, Chis.

Este panorama sobre el derecho a la salud y la situación de los pueblos indígenas nos plantea mirar y analizar qué posición tienen los sujetos que se encuentran construyendo otra forma de dar atención a la salud desde la práctica, otras veces desde la demanda, unas más desde la denuncia.

Desde hace tres décadas la atención a la salud se resaltó dentro de las demandas de los movimientos indígenas, durante el Primer Congreso Nacional Indígenas en 1974, donde participaron indígenas tsotsiles, tseltales, ch'oles y tojolabales, y donde se redacta la Declaración tsotsil de salud, cuyos contenidos versan sobre los siguientes puntos:

Medicina del doctor. Los médicos están concentrados en las ciudades y nunca salen al campo. Ignoran la lengua tsotsil, no conocen nuestras costumbres y sienten un gran desprecio por el indígena. En los centros de salud nos tratan mal, no hay comunicación posible con el doctor.

Los programas de salud no son realistas, pues no conocen nuestra costumbre y nunca tienen en cuenta la medicina de las yerbas. Un programa muy bien pensando que no lo lleva a efecto el personal preparado es inútil. 
La medicina es muy cara. Es un negocio. A veces solo tenemos dinero para pagar la ficha, pero no para la medicina. Se nos vende la medicina mala o ya pasada que es ineficaz. Por eso nosotros no confiamos en la medicina de los doctores. ${ }^{13}$

En 1994 aparece la salud como una de las principales demandas durante el levantamiento del Ejército Zapatista de Liberación Nacional, EZLN; 18 días después del levantamiento, en voz del subcomandante Marcos denuncian:

\begin{abstract}
...ंQuién tiene que pedir perdón y quién puede otorgarlo? ¿Los que, durante años y años, se sentaron ante una mesa llena y se saciaron mientras con nosotros se sentaba la muerte, tan cotidiana, tan nuestra que acabamos por dejar de tener miedo? ... ¿los muertos, nuestros muertos, tan mortales muertos de muerte «natural», es decir, de sarampión, tosferina dengue, cólera, tifoidea, mononucleosis, tétanos, pulmonía, paludismo y otras lindezas gastrointestinales y pulmonares? ¿Nuestros muertos tan mayoritariamente muertos, tan democráticamente muertos de pena porque nadie hacía nada, porque todos los muertos, nuestros muertos se iban así nomás, sin que nadie llevara la cuenta, sin que nadie dijera ¡YA BASTA!, que devolviera a esas muertes su sentido, sin que nadie pidiera a los muertos de siempre, nuestros muertos, que regresaron a morir otra vez pero ahora para vivir? ... ¿Quién tiene que pedir perdón y quién puede otorgarlo ${ }^{14}$
\end{abstract}

En 1997 promotores de diversas partes del país se reúnen y formulan la Declaración de Moisés Gandhi, donde se plantea la propia conceptualización de salud, a saber: «salud es vivir sin humillación», y se pronuncian por una salud en manos del pueblo.

- Para todos: la salud no es individual sino colectiva.

- Neutralidad: atender a todas las personas sin distinciones.

- Responsable: la salud debe estar en manos del pueblo. ${ }^{15}$

Diversas organizaciones de base en diferentes regiones indígenas han realizado un trabajo en salud desde finales de la década de 1970 y principios de 1980, muchas de ellas desde y con apoyo de las Comunidades Eclesiásticas de Bases, CEB, instituciones, organizaciones civiles e instituciones educativas. En esto me refiero a que en Chiapas desde hace más de treinta años se ha conformado una plataforma en salud en conjunto con la participación comunitaria a través de la formación de promotores de salud, así como un proceso organizativo por la demanda de sus derechos indígenas cuyo origen se 
encuentra precisamente en la inequidad, la desigualdad, el despojo, exclusión, discriminación y racismo que viven los pueblos originarios, pero cuya finalidad no solo ha estado en atender a la enfermedad sino principalmente en producir la vida.

Después del levantamiento zapatista, en Chiapas se fue construyendo y consolidando dentro de la propuesta de autonomía del EZLN el Sistema de Salud Autónomo Zapatista, SSAZ, que desde el inicio del movimiento ya contaba entre sus bases de apoyo con una cantidad importante de promotores y promotoras de salud, quienes se hicieron responsables de la atención asistencial de la población a partir de ese momento (Cuevas 2007: 3). Estos promotores y promotores fueron justamente los que anteriormente habían sido capacitados por diversas organizaciones e instituciones.

El SSAZ forma parte y está integrado en la estructura política y organización territorial del EZLN, siendo la instancia máxima las Juntas de Buen Gobierno, JBG, seguido de los Concejos Municipales Autónomos, donde existe una Coordinación de Salud integrada por representantes de promotores de salud y representantes de los comités de salud local; después están los Comités de Salud locales integrados por los promotores, vacunadores, promotores de salud reproductiva y personas de la comunidad.

Organizar un sistema propio además les ha permitido generar estrategias de negociación con un sentido de producir la vida con dignidad. Han generado vías para la canalización de pacientes al tercer nivel; para obtener de la Secretaría de Salud, SS, los medicamentos que necesitan enfermos de tuberculosis, así como las vacunas, cuya atención se basa en las necesidades de las mismas comunidades y desde una posición no subordinada.

Construir el sistema de salud autónomo ha implicado una posición de los sujetos como sujetos históricos, sociales y políticos, ejerciendo poder y sus derechos, ya que en la propia vivencia de otra visión de la salud están proporcionándose y construyendo las herramientas y estrategias para resistir a todas las formas a través de las cuales se ha buscado silenciarlos, desde donde han cuestionado y repensado el sentido de la salud, los derechos y la autonomía. 
Con todo, este fue un acercamiento al trabajo realizado, de ahí la importancia de resaltarlo, pues SSAZ no ha sido solo un proceso organizativo en salud, sino que está enmarcado en la propuesta de autonomía y el ejercicio de los derechos indígenas del movimiento zapatista. Lamentablemente, las investigaciones sobre el EZLN han versado más sobre la discusión de la autonomía, su organización política y su estructura de gobierno, dejando de lado el tema de la salud. La intención aquí es resaltar, aunque de manera muy esquemática, su importancia en el sentido de conocer cómo es que se organizan, cuál ha sido la percepción de la población en torno al servicio de salud autónomo, cómo se construye y permanece, y cómo fue su historia de constitución.

Son preguntas que en mi caso dejé pendiente, pero que seguro habrá más investigaciones que puedan profundizar en ello y darnos luz sobre los importantes aportes que este proceso plantea en la atención de la salud.

\section{A MANERA DE CONCLUSIÓN}

El reconocimiento, protección y garantía de los derechos humanos, incluido el derecho a la salud, a los pueblos indígenas tendrá que pasar por un cuestionamiento crítico al Estado y sus políticas sociales homogéneas y excluyentes, así como al discurso hegemónico de la política neoliberal de los derechos humanos; para entonces, los mismos pueblos y comunidades deben hacer de los derechos fundamentales realmente una bandera de lucha, más cotidiana, más operacional, planteada más desde las propias necesidades de los pueblos para la generación del buen vivir.

El Estado es central en el cumplimiento, garantía, protección y promoción del derecho a la salud, se ha comprometido además a garantizarlo al firmar una serie de convenios y pactos internacionales de derechos humanos, sin embargo, y para sortear el vacío entre lo que se da en la teoría y se hace en la práctica, será necesario el promover la exigibilidad y justiciabilidad del derecho a la salud, tanto entre los pueblos y comunidades como con la sociedad civil, tomadores de decisiones y planeadores y ejecutores de las políticas sociales, en aras de la construcción de una urgente política intersectorial en salud con la participación de los pueblos indígenas. 
Al mismo tiempo reconocer las alternativas y propuestas en torno a la atención de la salud, retomar planteamientos más amplios como son la autonomía y el ejercicio de los derechos desde los movimientos indígenas, organizaciones de médicos tradicionales, de base, de donde provienen las necesidades estratégicas de los pueblos.

Los retos por delante serán entonces identificar en estos movimientos y organizaciones los planteamientos de reivindicación de su propia capacidad emancipadora donde recuperen y fortalezcan sus prácticas colectivas que generen salud. Desde la perspectiva de los derechos humanos también reconocer los retos, como los que plantea Boaventura de Sousa Santos, abordarlos desde los movimientos sociales, realizar acciones ilegales y politizar las demandas y la lucha, para entonces así legalizar lo ahora ilegal a la luz del discurso de los derechos humanos. Para esto quizá antes se tendría que resolver la garantía de condiciones equitativas y justas para los pueblos. 


\section{BIBLIOGRAFÍA}

Abramovich y Courtis, 2003, «Apuntes sobre la exigibilidad judicial de los derechos sociales», en Derechos Sociales. Instrucciones de uso, compilado por Abramovich, V., M. J. Añón, Ch. Courtis, Doctrina Jurídica Contemporánea, México, pp. 55-78.

CDESC, 2000, Comité de Derechos Económicos, Sociales y Culturales, Observación general n. 14, «El derecho al disfrute más alto posible de salud», ONU.

Convenio 169, 1989, Convenio 169 Sobre Pueblos Indígenas y Tribales en países Independientes, OIT.

Cuevas, J. H., 2007, «Salud y Autonomía: el caso Chiapas», A case study commissioned by the Health Systems Knowledge Network, en http://www.who.int/social_determinants/resources/csdh_media/autonomy_mexico_200 7_es.pdf [consulta: septiembre de 2009].

Declaración de Alma Ata, 1978, «Conferencia Internacional sobre la Atención Primaria de la Salud, Alma-Alta», URSS, en http://www.paho.org/Spanish/dd/pin/almaata_declaracion.html [consulta: febrero de 2009].

Declaración de Moisés Gandhi, 1997, «Comunidad de Moisés Gandhi, Región Autónoma Toztz Choj», Chiapas, 24 de febrero, en http://www.ciepac.org/archivo/analysis/ decmoisesg.pdf [consulta: junio de 2010].

Lozoya, Xavier y Carlos Zolla (eds.), 1983, «Declaración tzotzil de la salud», en La medicina invisible. Introducción al estudio de la medicina tradicional de México, Folio Ediciones, México, D. F., pp. 3-5.

De Sousa Santos, Boaventura, 2004, «Los derechos Humanos y el Foro Social Mundial», en http://www.alainet.org [consulta: mayo de 2009]. 
Echeverri, María Esperanza, 2006, «Derecho a la salud. Estado y Globalización», Fac. Nac. Salud Pública, v. 24, número especial, en http://guajiros.udea.edu.co/fnsp/ Paginas/Congreso/ini/d9b.pdf [consulta: mayo de 2009].

Eibenschutz, Catalina, 2007, «Atención médica, neoliberalismo y reforma sanitaria en México», en La seguridad social: retos de hoy, coordinado por Raúl Molina S., UAM-I, México.

EZLN, 1994, Segunda Declaración de la Selva Lacandona, México, en http://es.wikisource.org/wiki/Segunda_Declaraci\%C3\%B3n_de_la_Selva_Lacandona [consulta: junio de 2009].

INEGI-OPS, 2000-2004, «Indiciadores de Salud, Instituto Nacional de Estadística y Organización Panamericana de la Salud», en http://sinais.salud.gob.mx/ indicadores/basicos.html [consulta: junio de 2010].

Informe de Organizaciones de la Sociedad Civil sobre la situación de los Derechos Económicos, Sociales, Culturales y Ambientales en México (1997-2006), «Informe Alternativo al IV Informe Periódico del Estado mexicano sobre la aplicación del PIDES», en http://www.equipopueblo.org.mx/descargas/ dcipu_mexicocoalition_Sp.pdf [consulta: junio de 2010].

Laurell, Asa Cristina, 1994, «Pronasol o la pobreza de los programas contra la pobreza», Nueva Sociedad, n. 131, pp. 156-170, en www.nuso.org/upload/ artículos/2339_1.pdf [consulta: mayo de 2010].

2010, «Revisando las políticas y discursos en salud en América Latina», en Medicinal Social, Salud para todos, v. 5, n. 1, en http://www.forosalud.org.pe [consulta: mayo de 2010]. 
Nájera Ortiz, Juan Carlos, 2005, «Seguimiento de pacientes con tuberculosis pulmonar en los Altos de Chiapas, México. Un análisis de casos de pacientes diagnosticados de 1998 a 2002», Tesis de maestría, El Colegio de la Frontera Sur, México.

Pérez, Mariana, 2010, «Sistema de protección a la salud», en Cinco Miradas del Derecho a la Salud. Estudios de caso en México, El Salvador y Nicaragua, coordinado por Mariana Pérez, Centro de Análisis e Investigación, FUNDAR, México.

PIDESC, 1966, Pacto de Derechos Económicos, Sociales y Culturales, ONU.

PHR, ECOSUR, CCESC, 2006, Pueblos excluidos, comunidades erosionadas. La situación del derecho a la salud en Chiapas, México, Physicians for Human Rigths/El Colegio de la Frontera Sur/Centro de Capacitación en Ecología y Salud para Campesinos, A.C., EUA.

Rivas Bocanegra, María Georgina, 1999, «Las organizaciones populares de salud en Las Margaritas, Chiapas. Sujeto Social en construcción», Tesis de maestría, UAM-X, México.

Sánchez Pérez, Héctor, 2007, «La salud enferma de Chiapas: consideraciones a 12 años de iniciado el conflicto armado», en Chiapas: la paz en la guerra, editado por Raúl Miranda Ocampo y Luz María Espinoza Cortés, Universidad Nacional Autónoma de México, El Colegio de la Frontera Sur, Comuna, México, pp. 287-323.

Sánchez Pérez, Héctor Javier y Guadalupe Vargas, 2008, «Salud Pública en Chiapas: tuberculosis pulmonar», en Pobreza y Salud: los retos de la investigación en Chiapas, en http://www.ecosur.mx/ecofronteras/ecofrontera/ecofront15/pdf/salud-publica.pdf [Consulta: junio de 2009].

Sánchez Pérez, H. J., M. Hernán, S. Hernández Díaz, J. M. Jansá, D. Halperin, A. Ascherio, 2002, «Detection of pulmonary tuberculosis in Chiapas, Mexico», Annals of Epidemiology, v. 12, n. 3, pp. 166-172. 
Schuftan, Claudio, 2007, «Un enfoque puntual de los principios y los temas clave de los derechos humanos aplicados a la salud», Revista de Medicina Social, v. 2, n. 2, pp. 7887, en http://journals.sfu.ca/socialmedicine/index.php/medicinasocial/rt/ [consulta: junio de 2009].

SSA, 1993, Norma Oficial para la prevención y control de la tuberculosis en la atención primaria a la salud, NOM-006-SSA2-1993, en http://www.salud.gob.mx/unidades/cdi/nom/m006ssa23.html [consulta: mayo de 2009].

Subcomandante Marcos, Carta del 18 de enero de 1994, «¿Quién tiene que pedir perdón y quién puede otorgarlo?», en http://videotecaalternativa.net/2010/11/11/\%C2\%BFdeque-tenemos-que-pedir-perdon-subcomandante-insurgente-marcos [consulta: junio de 2010]. 


\section{Notas}

\footnotetext{
${ }^{1}$ Resalto la importancia de la necesidad de realizar más análisis e investigación sobre lo que ha sido el SSAZ a través de los promotores, organizaciones que los han apoyado así como desde la misma población, ya que dichos estudios son aún muy escasos, o en el mejor de los casos es información valiosa con que cuentan las organizaciones civiles pero que todavía no se ha sistematizado.

${ }^{2}$ Véase Informe de Organizaciones de la Sociedad Civil sobre la situación de los Derechos Económicos, Sociales, Culturales y Ambientales en México (1997-2006). Informe Alternativo al IV Informe Periódico del Estado mexicano sobre la aplicación del PIDESC.

${ }^{3}$ Información del Centro de Derechos Humanos de la Montaña, Tlachinollan, disponible en la página http://www.tlachinollan.org/ [consulta: 4 de junio de 2010].

${ }^{4}$ Reporte sobre el proceso de devolución de la información del Informe Pueblos excluidos. Comunidades erosionadas. La situación del derecho a la salud en Chiapas, Documento interno, CCESC. Abril, 2007.

${ }^{5}$ En estas reuniones participaron diversas organizaciones que han trabajado el tema de la salud durante muchos años en diferentes regiones del estado, como Salud y Desarrollo Comunitario, SADEC, A. C.; Equipo de Apoyo en Salud y Educación Comunitaria, EAPSEC, A. C.; Centro de Asesoría Medio Ambiente y Defensoría del Derecho a la Salud, CAMADDS, A. C., Organizaciones Internacionales como Médicos del Mundo Francia y Médicos del Mundo Suiza; Médicos sin Fronteras.

${ }^{6}$ Durante este proceso quiero dar un agradecimiento a los promotores y promotoras que participaron en los talleres, especialmente a los coordinadores de la Clínica de Poza Rica, por su hospitalidad y entusiasmo en lo cotidiano para atender las injusticias reflejadas en la salud.

7 Este trabajo de capacitación se llevó a cabo con el apoyo de la Comisión Mexicana de Defensa y Promoción de los Derechos Humanos, CMDPDH, A. C., a través del abogado Mario Solórzano Betancourt en la participación durante los talleres con los promotores de salud de la región Selva.

${ }^{8}$ Testimonio de promotor de salud, región Selva. Febrero, 2008.

9 Promotores de la organización de las Cañadas. Relatorías de los talleres, Nuevo Poza Rica, Las Margaritas. Abril, 2008.

${ }^{10}$ Promotores de la organización de las Cañadas. Relatorías de los talleres, Nuevo Poza Rica, Las Margaritas. Abril, 2008.

11 Promotores de la organización de las Cañadas. Relatorías de los talleres, Nuevo Poza Rica, Las Margaritas. Abril, 2008.

12 Promotores de la organización de las Cañadas. Relatorías de los talleres, Nuevo Poza Rica, Las Margaritas. Abril, 2008.

13 Disponible en http://shelbijoseph.googlepages.com/Declaracintzotzilsobresalud.doc [consulta: 22 de junio de 2010].

14 Promotores de la organización de las Cañadas. Relatorías de los talleres, Nuevo Poza Rica, Las Margaritas. Abril, 2008.

15 Disponible en http:// shelbijoseph.googlepages.com/declaraciondemoisesgandhi.pdf [consulta: 22 de junio de 2010].
}

Fecha de recepción: 10 de diciembre de 2009.

Fecha de aceptación: 17 de agosto de 2010. 